\title{
Efficacy of Different IPM Modules against Melon Fruit Fly and American Serpentine Leaf Miner of Cucumber
}

\author{
S. A. Sarade, A. S. Bagde*, P. B. Mohite and V. M. Karade \\ Department of Entomology, R.C.S.M. College of Agriculture, Kolhapur- 416004 (MS), India \\ *Corresponding author
}

\section{A B S T R A C T}

Keywords

Cucumber, Fruit fly, Serpentine leaf minor

Article Info

Accepted:

12 August 2019

Available Online:

10 September 2019
Field experiment was conducted during Rabi season of 2017-18 with nine modules, replicated thrice, in the 'Randomized Block Design' with gross plot size $4.00 \mathrm{~m} \times 3.00 \mathrm{~m}$.As regards with melon fruit fly infestation, the module M7 (15.82 per cent) was found most effective at $1^{\text {st }}, 2^{\text {nd }}, 3^{\text {rd }}, 4^{\text {th }}$ and $5^{\text {th }}$ pickings. The next best module M4 (19.43 per cent) and M5 (22.12 per cent). The module M7 (23.82 per cent) was found most superior in reducing American serpentine leaf miner population at 25, 45 and 65 days after sowing. Followed by module M4 (25.80 per cent) and M5(28.74 per cent).

\section{Introduction}

Cucumber, (Cucumis sativus L.), which is one of the monocecious annual crops of the cool climate belongs to the cucurbitacaceae family comprising 118 genera and 825 species.

Fruit fly, B. cucurbitae is one of the most destructive pests often rendering cultivation of cucumber unprofitable.

The fruit are damaged by the maggots of this fly as the female fly lays it eggs in the tissues of fruits. The maggots feed on flesh and look like rotten fruits. Hence, present investigation was undertaken to study the efficacy of different IPM modules against melon fruit fly and American serpentine leafminer of cucumber under natural infestation in the field conditions.

\section{Materials and Methods}

The field experiments was conducted during Rabi season of 2017-18 with local Kheera variety of cucumber with Nine treatments replicated Thrice in the 'Randomized Block Design' with gross plot size $4.00 \mathrm{~m} \times 3.00 \mathrm{~m}$ carried out during Rabi 2017-18 at Post Graduate Research Farm, Department of Horticulture, RCSM College of Agriculture, Kolhapur. 


\section{Method of recording observations}

The observations of fruit fly damage were recorded at weekly interval throughout the crop season.

The damaged and healthy fruits were recorded at each picking for recording fruit infestation by fruit flies. . (Dubale et al., 2018)

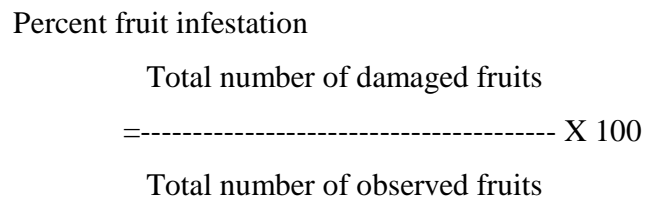

Observations on per cent damaged leaves by American leaf miner were recorded on randomly selected three plants in each plot for these purpose five plants per plot were tagged.

The observations were recorded by counting total number of leaves per plant and number of leaf miner infested leaves. The per cent damage of leaves was expressed as below.

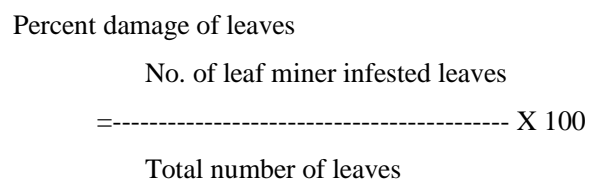

Total number of leaves

\section{Statistical analysis}

In order to compare the treatment effect based on generated data of field experiments, the natural counts were subjected to transformation as per statistical methods suggested by Panse and Sukhatme, (1967).

The data on pest infestation based on calculated percentage were transformed to arc sine values in respect of melon fruit fly, e and serpentine leaf miner infestation.

\section{Results and Discussion}

Efficacy of different modules against fruit infestation by melon fruit fly (B. cucurbitae Coq.) of cucumber

Data pertaining to the survival population of melon fruit fly of cucumber on number basis at each picking are presented in Table 2. All the modules were found to be significantly superior in reducing population of melon fruit fly when observations were recorded at $1^{\text {st, }}$ $2^{\text {nd, }} 3^{\text {rd }}, 4^{\text {th }}, 5^{\text {th }}$, picking.

From the overall performance of all the modules it was found that all the modules were significantly superior over control in reducing melon fruit fly infestation. The module M7 (15.82 per cent) was found as the best treatment with 56.26 per cent reduction over control. The next promising modules in decreasing order were M4 (19.43 per cent), M2 (22.12 per cent), M6 (24.76 per cent), M5 (27.15 per cent) and M1 (29.66 per cent) which are found equally effective in next order of efficacy. The module M3 (31.51 per cent) and M8 (33.49 per cent) were least effective.

These results are confirmative with result of Golvankar et al.,(2018) the results on efficacy of insecticides against fruit flies infesting cucumber indicated that spinosad 45 SC @ 0.014 per cent was the best treatment which recorded minimum (15.38\%) mean fruit infestation and was at par with emamectin benzoate 5 SG @ 0.002 per cent (20.49\%).

\section{Efficacy of different modules against American serpentine leaf miner ( $L$. trifolii Burgess) of cucumber}

Data pertaining to the survival population of leaf miner of cucumber on number basis at each picking are presented in Table 3. 
Table.1 Module details for pest complex of cucumber

\begin{tabular}{|c|c|}
\hline Sr.No. & Module Details \\
\hline M1. & $\begin{array}{l}\text { Soil application of Carbofuran 10\%G @ } 15 \mathrm{Kg} / \mathrm{ha}+\text { erection of yellow sticky traps }(1-2 \text { traps } \\
\left.@ 50-100 \mathrm{~m}^{2}\right)+ \text { foliar spray of NSE 5\% + spaying of Indoxacarb } 14.55 \% \mathrm{SC} @ 0.5 \mathrm{ml} / \mathrm{L} .\end{array}$ \\
\hline M2. & $\begin{array}{l}\text { Soil application of Trichoderma viridae @ } 3-5 \mathrm{Kg} / \mathrm{ha}+\text { poison bait(Malathion } 20 \mathrm{ml}+20 \mathrm{~L} \text { water } \\
+50 \mathrm{~g} \text { molasses) + Metarhizium anisopliae @ } 5 \mathrm{~g} / \mathrm{L}+\text { foliar spray of Deltametrin } 1.8 \% \mathrm{EC} @ \\
1.25 \mathrm{ml} / \mathrm{L} \text {. }\end{array}$ \\
\hline M3. & $\begin{array}{l}\text { Spaying of Pseudomonas fluorescens @ } 4-6 \mathrm{~g} / \mathrm{L}+\text { erection of Cue lure trap + spraying of } \\
\text { Lecanicilicium laccani @ 5g/L + foliar spray of Propergite } 57 \% \mathrm{EC} @ 2 \mathrm{ml} / \mathrm{L} \text {. }\end{array}$ \\
\hline M4. & $\begin{array}{l}\text { Spraying of Spirotetramate } 15 \% \mathrm{OD} @ 1 \mathrm{ml} / \mathrm{L}+\text { foliar spray of NSE } 5 \% \text { + spraying of Beauveria } \\
\text { bassiana @ 5g/L + spraying of Flubendamide } 39.35 \% \text { SC @ 0.1ml/L. }\end{array}$ \\
\hline M5. & $\begin{array}{l}\text { Trap crop Marigold (Two rows) + spraying of Flonicamide 50\%WG @ 0.3gm/L + spraying of } \\
\text { Metarhizium anisopliae@ } 5 \mathrm{~g} / \mathrm{L}+\text { foliar spray of Chloranatriniprole18.5\%SC @ 0.25ml/L. }\end{array}$ \\
\hline M6. & $\begin{array}{l}\text { Trap crop French bean (Two rows) + spraying of Dinotefuron } 20 \% \mathrm{SG} @ 0.25 \mathrm{~g} / \mathrm{L}+\text { foliar spray } \\
\text { of NSE } 5 \%+\text { spraying of Abamectin } 1.9 \% \text { EC } 0.5 \mathrm{ml} / \mathrm{L} \text {. }\end{array}$ \\
\hline M7. & $\begin{array}{l}\text { Trap crop Maize (Two rows) + spraying of Indoxacarb } 14.55 \% \mathrm{SC} @ 0.5 \mathrm{ml} / \mathrm{L}+\text { spraying of } \\
\text { Metarhizium anisopliae@ } 5 \mathrm{~g} / \mathrm{L}+\text { spraying of NSE } 5 \% .\end{array}$ \\
\hline M8. & $\begin{array}{l}\text { Trap crop Mustard (Two rows) + soil application of Phorate 10\%G @ } 15 \mathrm{Kg} / \mathrm{ha}+\text { spraying of } \\
\text { Metarhizium anisopliae@ } 5 \mathrm{~g} / \mathrm{L}+\text { spraying of Acephate } 75 \% \mathrm{SP} @ 0.5 \mathrm{~g} / \mathrm{L} .\end{array}$ \\
\hline M9. & Untreated control. \\
\hline
\end{tabular}


Table.2 Efficacy of different modules against melon fruit fly on cucumber

\begin{tabular}{|c|c|c|c|c|c|c|c|}
\hline \multirow{2}{*}{ Module } & \multicolumn{6}{|c|}{ Percent fruit infestation at different pickings } & \multirow{2}{*}{$\begin{array}{l}\text { Per cent } \\
\text { Reduction } \\
\text { over } \\
\text { control }\end{array}$} \\
\hline & $1^{\text {st }}$ Picking & $\begin{array}{l}2^{\text {nd }} \\
\text { Picking }\end{array}$ & $\begin{array}{l}3^{\text {rd }} \\
\text { Picking }\end{array}$ & $\begin{array}{l}4^{\text {th }} \\
\text { Picking }\end{array}$ & $\begin{array}{l}5^{\text {th }} \\
\text { Picking }\end{array}$ & Mean & \\
\hline M1 & $\begin{array}{l}28.11 \\
(32.09)^{*}\end{array}$ & $\begin{array}{l}30.33 \\
(33.42)\end{array}$ & $\begin{array}{l}31.96 \\
(34.43)\end{array}$ & $\begin{array}{l}28.88 \\
(32.51)\end{array}$ & $\begin{array}{l}29.02 \\
(32.60)\end{array}$ & $\begin{array}{l}29.66 \\
(33.00)\end{array}$ & 18.00 \\
\hline M2 & $\begin{array}{l}20.88 \\
(27.19)\end{array}$ & $\begin{array}{l}23.14 \\
(28.75)\end{array}$ & $\begin{array}{l}23.97 \\
(29.31)\end{array}$ & $\begin{array}{l}21.72 \\
(27.78)\end{array}$ & $\begin{array}{l}20.89 \\
(27.20)\end{array}$ & $\begin{array}{l}22.12 \\
(28.06)\end{array}$ & 38.84 \\
\hline M3 & $\begin{array}{l}29.96 \\
(33.19)\end{array}$ & $\begin{array}{l}32.62 \\
(34.83)\end{array}$ & $\begin{array}{l}33.21 \\
(35.19)\end{array}$ & $\begin{array}{l}31.11 \\
(33.90)\end{array}$ & $\begin{array}{l}30.64 \\
(33.61)\end{array}$ & $\begin{array}{l}31.51 \\
(34.15)\end{array}$ & 12.88 \\
\hline M4 & $\begin{array}{l}18.21 \\
(25.26)\end{array}$ & $\begin{array}{l}19.86 \\
(26.46)\end{array}$ & $\begin{array}{l}20.7 \\
(27.06)\end{array}$ & $\begin{array}{l}19.97 \\
(26.54)\end{array}$ & $\begin{array}{l}18.42 \\
(25.42)\end{array}$ & $\begin{array}{l}19.43 \\
(26.16)\end{array}$ & 46.28 \\
\hline M5 & $\begin{array}{l}25.96 \\
(30.63)\end{array}$ & $\begin{array}{l}28.73 \\
(32.41)\end{array}$ & $\begin{array}{l}29.11 \\
(32.65)\end{array}$ & $\begin{array}{l}26.76 \\
(31.15)\end{array}$ & $\begin{array}{l}25.17 \\
(30.11)\end{array}$ & $\begin{array}{l}27.15 \\
(31.40)\end{array}$ & 24.94 \\
\hline M6 & $\begin{array}{l}23.38 \\
(28.92)\end{array}$ & $\begin{array}{l}26.08 \\
(30.71)\end{array}$ & $\begin{array}{l}26.86 \\
(31.22)\end{array}$ & $\begin{array}{l}24.37 \\
(29.58)\end{array}$ & $\begin{array}{l}23.11 \\
(28.73)\end{array}$ & $\begin{array}{l}24.76 \\
(29.84)\end{array}$ & 31.55 \\
\hline M7 & $\begin{array}{l}14.08 \\
(22.039)\end{array}$ & $\begin{array}{l}16.46 \\
(23.94)\end{array}$ & $\begin{array}{l}17.86 \\
(25.00)\end{array}$ & $\begin{array}{l}16.11 \\
(23.66)\end{array}$ & $\begin{array}{l}14.57 \\
(22.44)\end{array}$ & $\begin{array}{l}15.82 \\
(23.43)\end{array}$ & 56.26 \\
\hline M8 & $\begin{array}{l}31.88 \\
(34.38)\end{array}$ & $\begin{array}{l}34.58 \\
(36.02)\end{array}$ & $\begin{array}{l}35.06 \\
(36.31)\end{array}$ & $\begin{array}{l}33.46 \\
(35.34)\end{array}$ & $\begin{array}{l}32.46 \\
(34.73)\end{array}$ & $\begin{array}{l}33.49 \\
(35.36)\end{array}$ & 7.41 \\
\hline $\begin{array}{l}\text { M9. } \\
\text { Untreated } \\
\text { control }\end{array}$ & $\begin{array}{l}33.51 \\
(35.37)\end{array}$ & $\begin{array}{l}37.11 \\
(37.53)\end{array}$ & $\begin{array}{l}38.08 \\
(38.10)\end{array}$ & $\begin{array}{l}37.03 \\
(37.48)\end{array}$ & $\begin{array}{l}35.11 \\
(36.34)\end{array}$ & $\begin{array}{l}36.17 \\
(36.97)\end{array}$ & \\
\hline S.E. \pm & 1.15 & 0.94 & 0.89 & 1.01 & 0.86 & & \\
\hline C.D. $(5 \%)$ & 3.46 & 2.83 & 1.26 & 3.05 & 2.60 & & \\
\hline $\mathbf{C V}$ & 7.92 & 5.89 & 5.41 & 6.60 & 5.88 & & \\
\hline
\end{tabular}

DAS $=$ Days after sowing

*Figures in parentheses are arcsine transformed value 
Table.3 Efficacy of different modules against serpentine leaf miner on cucumber

\begin{tabular}{|c|c|c|c|c|c|c|}
\hline \multirow[t]{2}{*}{ Module } & \multicolumn{5}{|c|}{ Percent leaf mines infestation/plant } & \multirow{2}{*}{$\begin{array}{l}\text { Per cent } \\
\text { Reduction } \\
\text { over control }\end{array}$} \\
\hline & Pre count & 25 DAS & 45 DAS & 65 DAS & Mean & \\
\hline M1 & $\begin{array}{l}41.19 \\
(39.93)^{*}\end{array}$ & $\begin{array}{l}32.5 \\
(34.76)\end{array}$ & $\begin{array}{l}26.3 \\
(30.85)\end{array}$ & $\begin{array}{l}29.31 \\
(32.78)\end{array}$ & $\begin{array}{l}32.33 \\
(34.65)\end{array}$ & 9.16 \\
\hline M2 & $\begin{array}{l}42.63 \\
(40.76)\end{array}$ & $\begin{array}{l}31.38 \\
(34.07)\end{array}$ & $\begin{array}{l}19.32 \\
(26.07)\end{array}$ & $\begin{array}{l}21.64 \\
(27.72)\end{array}$ & $\begin{array}{l}28.74 \\
(32.42)\end{array}$ & 19.25 \\
\hline M3 & $\begin{array}{l}42.98 \\
(40.96)\end{array}$ & $\begin{array}{l}26.67 \\
(31.09)\end{array}$ & $\begin{array}{l}30.65 \\
(33.62)\end{array}$ & $\begin{array}{l}33.47 \\
(35.35)\end{array}$ & $\begin{array}{l}33.44 \\
(35.33)\end{array}$ & 6.04 \\
\hline M4 & $\begin{array}{l}40.32 \\
(39.42)\end{array}$ & $\begin{array}{l}27.81 \\
(31.83)\end{array}$ & $\begin{array}{l}16.1 \\
(23.66)\end{array}$ & $\begin{array}{l}18.98 \\
(25.83)\end{array}$ & $\begin{array}{l}25.80 \\
(30.53)\end{array}$ & 27.51 \\
\hline M5 & $\begin{array}{l}42.6 \\
(40.74)\end{array}$ & $\begin{array}{l}32.53 \\
(34.77)\end{array}$ & $\begin{array}{l}24.76 \\
(29.84)\end{array}$ & $\begin{array}{l}27.27 \\
(31.48)\end{array}$ & $\begin{array}{l}31.79 \\
(34.32)\end{array}$ & 10.68 \\
\hline M6 & $\begin{array}{l}42.94 \\
(40.94)\end{array}$ & $\begin{array}{l}32.26 \\
(34.61)\end{array}$ & $\begin{array}{l}21.43 \\
(27.58)\end{array}$ & $\begin{array}{l}24.24 \\
(29.49)\end{array}$ & $\begin{array}{l}30.22 \\
(33.35)\end{array}$ & 15.09 \\
\hline M7 & $\begin{array}{l}40.35 \\
(39.44)\end{array}$ & $\begin{array}{l}26.34 \\
(30.88)\end{array}$ & $\begin{array}{l}12.91 \\
(21.06)\end{array}$ & $\begin{array}{l}15.67 \\
(23.32)\end{array}$ & $\begin{array}{l}23.82 \\
(29.21)\end{array}$ & 33.07 \\
\hline M8 & $\begin{array}{l}41.17 \\
(39.91)\end{array}$ & $\begin{array}{l}33.63 \\
(35.44)\end{array}$ & $\begin{array}{l}29.71 \\
(33.03)\end{array}$ & $\begin{array}{l}33.44 \\
(35.33)\end{array}$ & $\begin{array}{l}34.49 \\
(35.96)\end{array}$ & 3.09 \\
\hline $\begin{array}{l}\text { Untreated } \\
\text { control }\end{array}$ & $\begin{array}{l}41.24 \\
(39.95)\end{array}$ & $\begin{array}{l}34.94 \\
(36.23)\end{array}$ & $\begin{array}{l}31.76 \\
(34.30)\end{array}$ & $\begin{array}{l}34.4 \\
(35.91)\end{array}$ & $\begin{array}{l}35.59 \\
(36.62)\end{array}$ & \\
\hline S.E. \pm & NS & 0.82 & 1.10 & 0.87 & - & \\
\hline C.D.(5\%) & NS & 2.48 & 3.30 & 2.60 & & \\
\hline CV & & 5.40 & 7.45 & 6.18 & & \\
\hline
\end{tabular}

DAS= Days after sowing

*Figures in the parentheses are arcsine transformed value

All the modules were found to be significantly superior in reducing population of leaf miner when observations were recorded at 25,45,65 DAS.

From the overall performance of all the modules it was found that all the modules were significantly superior over control in reducing per cent leaf miner infestation.

The module M7 (23.82 per cent) was found as the best treatment with 33.07 per cent reduction over control. The next promising modules in decreasing order were M4 (25.80 per cent), M2 (28.74per cent) and M6 (30.22 per cent) which are found equally effective in next order of efficacy.

The module M5 (31.79 per cent) was found least effective. These results are confirmative with the results Desai (2018) revealed that on efficacy of different insecticides against $L$. trifolii through foliar sprays. The abamectin $1.9 \mathrm{EC}$ at $0.00057 \%$ was most effective treatment for suppression Liriomyza trifolii and it was followed by cartap hydrochloride $50 \mathrm{SP}$ at $0.05 \%$. 


\section{References}

Dubale, M.M., Jalgaonkar, V. N., Golvankar K. V. and Munj A. Y. 2018. Studies on incidence of fruit flies in relation to weather parameters parameters on ridge gourd in Konkan region. $J$. of Entomology and Zoology Studies 2018: 6(5): 2012-2014.

Desai, N. R., C. S. Bhoge, D. B. Pawar and R. S. Bhoge.2018. Bioefficacy of Different Insecticides against Leaf Minor (Liriomyza trifolii) on Cucumber and Their Effect on Natural
Enemies. Int.J.Curr.Microbiol.App.Sci (2018) Special Issue-6: 2392-2395 2392

Golvankar, G., Naik, K. V., Shinde, P. B., ang Jaldaonkar, V. N. 2018. Bio-efficacy of insecticides against fruit flies infesting cucumber. Int. J. of Chemical Stuidies 2018; 6(5): 1681-1684.

Panse, V. G. and Sukhatme, P. V. 1967. Statistical Method for Agricultural Workers. Second edition. Indian Council of Agricultural Research. New Delhi, 38 1p.

\section{How to cite this article:}

Sarade, S. A., A. S. Bagde, P. B. Mohite and Karade, V. M. 2019. Efficacy Of Different IPM Modules against Melon Fruit Fly and American Serpentine Leaf Miner of Cucumber. Int.J.Curr.Microbiol.App.Sci. 8(09): 1240-1245. doi: https://doi.org/10.20546/ijcmas.2019.809.142 